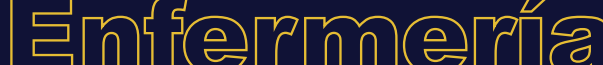 \\ In I versitaría
}

\section{Importancia de la calidad de la Investigación Cualitativa}

\section{The importance of quality in Qualitative Research}

\section{Importância da qualidade da Pesquisa Cualitativa}

El uso creciente de la Investigación Cualitativa (IC), por parte de enfermeras investigadoras en el mundo, desde los años noventa, ha llevado a que poco a poco sea reconocida como una herramienta legítima para la comprensión de las perspectivas de las personas y los sujetos sociales, de manera particular los sujetos del cuidado de enfermería. Así, a través de la práctica de la IC, que partió de una crítica a la hegemonía de los modelos biologicistas y positivistas, con el interés de contribuir a la humanización del cuidado de enfermería, algunas enfermeras en América Latina, han buscado incluir las voces de las personas con quienes trabajamos como parte del conocimiento disciplinar, como insumos para el diseño de políticas y acciones en el campo de la salud ${ }^{1,2}$.

De los cursos sobre IC con estudiantes de enfermería y de otras disciplinas, recuerdo que la mayoría de las veces, cuando llegaba al punto en el que había cierta claridad sobre la IC, como una forma de conocer las perspectivas y voces de las personas, alguien intervenía con energía diciendo una frase como: pero, eso tiene sesgo porque es muy subjetivo. Luego nos tomaba un buen tiempo de la clase, profundizar y distinguir que el hecho de trabajar sobre las realidades subjetivas, no significaba que los datos son erróneos o que la IC no sigue criterios de calidad. Estas preocupaciones y cuestionamientos sobre una supuesta debilidad y el riesgo de la baja calidad de la IC por el hecho de estar hecha a partir de datos subjetivos, han sido expuestos también por investigadoras en enfermería y revistas en el campo de la salud ${ }^{3,4}$

Sin embargo, los riesgos que pueden afectar la calidad de la IC no se encuentran en trabajar con las realidades subjetivas, ni en utilizar al investigador como principal herramienta, para la comprensión e interpretación de las perspectivas de los participantes en la investigación. Por el contrario, la subjetividad de los participantes y la reflexividad del investigador son características propias de la IC que, manejadas con rigor, fortalecen su calidad. La amenaza real a una IC de calidad se encuentra en lo que diversos estudiosos hemos planteado, como motivaciones equivocadas, deficiencias en la formación de los investigadores y carencias de criterios de calidad en los diferentes momentos de las investigaciones.

En cuanto a los investigadores, son diversos los argumentos que he escuchado y leído a colegas y estudiantes, sobre las razones por las que escogieron a la IC como herramienta metodológica. Si bien, algunos motivos se relacionan con su interés en comprender las perspectivas de las personas con quienes trabajamos y en ser la voz de ellos, otras la ven como una moda interesante que les da cierto prestigio y los coloca por encima de colegas menos actualizados. También, hay quienes la eligen porque, al no tener formación y con un limitado conocimiento, reducen la IC a la recolección de datos, de manera equivocada creen que es más fácil. Luego cuando se enfrentan al análisis, surgen obstáculos que difícilmente pueden sobrepasar. Desafortunadamente, hay quienes optan por desconocer las tradiciones y los saberes sobre el análisis cualitativo, así como la relación de la IC con las teorías y las tradiciones de los temas estudiados. 
Estas carencias, llevan a que haya investigadores que presentan informes o artículos con análisis que se reducen a un recuento de testimonios o interpretaciones desarticuladas y descontextualizadas. De esta manera, le quitan a la IC su verdadera fuerza que está en la construcción de interpretaciones enraizadas en las perspectivas de los actores sociales y contextualizadas, en las teorías y las realidades sociales.

Al igual que la investigación de corte cuantitativo, la práctica de la IC requiere que como investigadoras realicemos de manera juiciosa, consciente y rigurosa, acciones orientadas a garantizar interacciones éticas, respetuosas y amables con los participantes, al mismo tiempo, calidad de los datos recolectados y de nuestras interpretaciones. Si bien, actualmente hay una amplia diversidad de posturas y perspectivas sobre cuáles serían unos criterios de calidad aceptables ${ }^{5}$, hay un acuerdo amplio tanto en la IC en ciencias sociales, como en el campo de la salud y de la enfermería, sobre la importancia y la necesidad de tener y seguir criterios de calidad. Esto significa, seguir lineamientos y realizar acciones orientadas a lograr validez y confiabilidad en todos los momentos de la investigación.

En este contexto, además de reconocer que las discusiones entre la diversidad de perspectivas forman parte de la realidad académica de la IC, considero que siguen teniendo vigencia los planteamientos de Miles y Huberman, en un libro clásico de análisis cualitativo ${ }^{5}$, sobre la credibilidad, la autenticidad, la plausibilidad y la verosimilitud, como características de una IC de calidad. Asimismo, el sentido y la calidad de los datos y las interpretaciones de los investigadores pueden ser valorados a partir de la validez interna, es decir, de la adecuación de sus narrativas e interpretaciones con la realidad de las perspectivas planteadas por los participantes, con preguntas como:

- ¿Los resultados parecen ser coherentes y consistentes entre sí?

- ¿Los juicios y conclusiones de la investigadora son coherentes con las descripciones que presenta?

- ¿Las personas que dan la información son informantes confiables? Es decir, ¿son conocedores y han vivido las realidades que son tema de la investigación?

- ¿Las descripciones son detalladas y ricas?

- ¿La investigadora describe detalladamente cómo se hizo la investigación, los métodos, las limitaciones, los problemas, las fuentes de financiación? ${ }^{6}$

Asimismo, si queremos que nuestra investigación sea tomada en serio y en cuenta como fuente para el diseño de políticas y acciones que mejoren la salud de las personas con quienes trabajamos, debemos buscar la validez en todos los pasos del proceso investigativo, de manera particular a través de:

- Dedicar tiempo suficiente para lograr el conocimiento y la construcción de confianza, con las personas a quienes queremos invitar a participar en la investigación. Esto implica dedicar tiempo en el campo y a conocer los contextos en los que viven.

- Realizar un proceso de invitación participativa a los posibles participantes.

- Realizar con cada uno de los participantes un proceso de consentimiento informado detallado y completo, que cumpla con los requisitos éticos y legales vigentes.

- Utilizar herramientas de recolección de datos que garanticen conocer de manera amplia y profunda las perspectivas de los participantes, que se ajusten a sus circunstancias particulares, como, por ejemplo, edad, procedencia urbana rural, etnia, género y escolaridad. Esto significa también valorar la utilidad y la pertinencia de utilizar y combinar diversas técnicas de datos verbales, visuales y digitales.

- Es recomendable llevar registros de diarios de campo.

- Definir lineamientos y criterios de calidad para realizar las transcripciones.

- Seguir pasos para el análisis y la interpretación, que incluyan de manera diferenciada al menos un momento descriptivo y otro analítico-interpretativo.

- Reconocer las características del análisis cualitativo, de manera particular la construcción inductiva a partir de las voces de los participantes. Igualmente, significa reconocer que, a diferencia del análisis cuantitativo, en el que la teoría es un punto de partida para definir hipótesis que luego voy a medir, en el análisis cualitativo, la teoría es germen de categorías deductivas iniciales, fuente a la 
que vuelvo durante los diferentes momentos de la investigación, con el fin de contrastar, conversar y construir las interpretaciones.

Aunque la validez externa, entendida como la posibilidad de transferir las conclusiones de la investigación a otros contextos, no es una característica ni una intención necesaria de la IC, es posible buscar generalizaciones a partir del poder conceptual, es decir, con base en las relaciones que encuentro relevantes entre las interpretaciones de las voces y perspectivas de los participantes en mi investigación, con la o las teorías sobre el tema del estudio ${ }^{6}$.

Otras herramientas que promueven la calidad de la IC, las encuentro en la reflexividad, la triangulación y la inclusión de los participantes, así como de otros investigadores en el análisis y la construcción de las interpretaciones. A través de la reflexividad, reconocemos de manera crítica la relación de nuestros intereses, conocimientos y antecedentes personales, con la investigación y las interpretaciones que construimos. La triangulación, entendida como una técnica que combina dos o más perspectivas, modelos o métodos, o diferentes fuentes de información, tiene la capacidad, ya sea por complementariedad o por contradicción, de dar mayor precisión, alcance y riqueza al análisis ${ }^{7}$.

Finalmente, promovemos la calidad manteniendo una conversación con los participantes y otros investigadores. A través de medios como; compartir, discutir y ajustar informes parciales e informes finales, cumplimos con el requisito ético y humano de retribuir y agradecer, a quienes de manera generosa nos ayudaron en nuestra investigación. Al mismo tiempo, ellos nos pueden ir contando sobre cómo se sienten representados, sobre la riqueza y la calidad de las descripciones y narrativas, sobre las interpretaciones que dan a los datos contradictorios que hemos ido encontrando. Asimismo, otros investigadores que puedan ejercer, no como jueces, sino como pares-amigos pueden ser fuente de gran riqueza al darnos la oportunidad de cuestionar, ampliar nuestras perspectivas y reconocer nuevas relaciones o interpretaciones. En este contexto, de manera semejante a la representación de la IC como un tejido realizado por diferentes manos, la calidad de la IC la construimos de manera conjunta participantes, investigadores y colegas, con diversas herramientas en todos los momentos de la investigación.

\section{Referencias}

1. Duque-Páramo MC, Castellanos-Soriano F, Buitrago-García DC. La investigación cualitativa en enfermería en Colombia (1990-2010). Enfermería glob. 2014; 13(36): 208-22.

2. Duque-Páramo MC, Padilha MI, Sanhueza-Alvarado OI, Alonso-Castillo MM, Castellanos-Soriano F, López-García KS, et al. Qualitative nursing research in Latin America: the cases of Brazil, Chile, Colombia and Mexico. En: Beck CT, (ed.). Routledge International Handbook of Qualitative Nursing Research. London: Routledge; 2013. https://doi.org/10.4324/9780203409527

3. Benjumea CC. La investigación cualitativa y el desarrollo del conocimiento en enfermería. Texto contexto enferm. 2010; 19(4): 762-6.

4. PLoS Medicine Editors. Qualitative research: understanding patients' needs and experiences. PLoS Med. 2007; 4(8): e258. https://doi.org/10.1371/journal.pmed.0040258

5. Walby K, Luscombe A. Criteria for quality in qualitative research and use of freedom of information requests in the social sciences. Qual Res. 2016; 17(5): 537-53.

https://doi.org/10.1177/1468794116679726

6. Miles MB, Huberman AM. Qualitative data analysis: an expanded sourcebook. $2^{\text {nd }}$ ed. Thousand Oaks: Sage Publications; 1994. xiv, p.338.

7. Taylor C, Gibbs GR, Lewins A. Quality of qualitative analysis. ONELINE QDA. Huddersfield, England: University of Huddersfield; 2005. [Actualización 29 noviembre 2011; Consultado marzo 4 2019]. Disponible en: https://bit.ly/2YZVUf3

M.C. Duque-Páramo

Investigador y Consultor Independiente 